\title{
LA AUDITORÍA INTEGRAL COMO HERRAMIENTA DE VALIDACIÓN DE LA GESTIÓN INSTITUCIONAL
}

\section{The integral audit as a validation tool for institutional management}

\section{Marcos Eduardo Cantos Ochoa*}

\section{RESUMEN}

La auditoría integral como evaluación preventiva, permite validar los sistemas administrativos, financiero, de cumplimiento y de gestión que gobiernan una entidad, con el objeto de proponer alternativas para el logro adecuado de sus fines y/o el mejor aprovechamiento de sus recursos. Basado en las Normas Internacionales de Auditoría, Normas de Auditoría Generalmente Aceptadas, Normas de Auditoría Gubernamental, Normas Técnicas de Control Interno para el sector Público entre otras y la revisión documental de autores reconocidos en el área como Blanco (2012a, 2012b), Franklin (2007) y Mantilla (2005), el estudio analiza el proceso de ejecución de la auditoría con el enfoque por analogía de las revisiones financieras, de cumplimiento, control interno y de gestión, desarrollada en una institución pública dedicada al deporte del Cantón Cañar, Provincia Cañar - Ecuador, en el período fiscal 2016, para facilitar una visión del conjunto de responsabilidades más importantes de la gestión institucional. Metodológicamente se aplicó una auditoría integral, para analizar aquellos procedimientos de control implementados por todos los miembros de la organización, en relación con los componentes del sistema COSO, aplicando las preguntas del cuestionario al Presidente, Secretario, Tesorero y Jefe de Talento Humano; examinar el grado de cumplimiento de la normativa aplicable; el proceso financiero y la gestión a través de indicadores. Los resultados demuestran que la Auditoría Integral es la evaluación multidisciplinaria, independiente y con enfoque de sistemas, del grado y forma de cumplimiento de los objetivos de una institución, de la relación con su entorno, así como de sus operaciones. Se hace énfasis en su importancia como herramienta gerencial, para la toma de decisiones y para poder verificar los puntos débiles de las organizaciones con el fin de tomar medidas y precauciones a tiempo.

\footnotetext{
* Docente de la Unidad Académica de Administración de la Universidad Católica de Cuenca. Magister en Auditoría Integral por la Universidad Técnica Particular de Loja, Ecuador. Ingeniero Empresarial por la Universidad Católica de Cuenca. Cursante del tercer ciclo del Doctorado en Ciencias Sociales mención Gerencia de la Uni versidad del Zulia. Cuenca, Ecuador. Correo electrónico: mecantoso@ucacue.edu.ec
} 
Palabras claves: auditoría integral, control interno, operaciones financieras, gestión administrativa.

\section{ABSTRACT}

The integral audit as preventive evaluation, allows validating the administrative, financial, compliance and, management systems that govern an entity, in order to purpose alternatives for the adequate achievement of its purposes and the best use of its resources. Based on international auditing standards, generally acceptable auditing standards, Government audit standards, technical standards of internal control for the public sector among others and the documentary review of recognized authors in the area such as: Blanco (2012a, 2012b), Franklin (2007) y Mantilla (2005), The study analyzes the process of execution of the audit with the approach by analogy of the financial reviews of compliance internal control and management, developed in a public institution dedicated to sports of the Cantón Cañar, Province of Cañar - Ecuador, in the fiscal year 2016 to facilitate a vision of the set of most important responsibilities of institutional management. Methodologically, a comprehensive audit was applied to analyze those control procedures implemental by all the members of the organization in relation to the components from the system COSO, applying the questions of the questionnaire to the President, Secretary, Treasurer and Head of Human Talent; examine, the degree of compliance with applicable regulations, the financial process and management through indicators. The results show that the integral audit is the independent and multidisciplinary evaluation with systems approach of the degree and form of fulfillment of the objectives of an institution, of the relation with its surroundings as well as its operations. Emphasis is placed on its importance as a management tool for decision making and to verify the weak points of organizations in order to take timely measures and precautions.

Key Words: Integral Audit, Internal Control, Financial Operations, Administrative management

\section{Introducción}

Las bases conceptuales tradicionales relativas al desarrollo de auditorías o exámenes especiales financieros, no abarcan otras labores esenciales de la organización y reflejan un análisis incompleto de sus operaciones. Esta situación, amerita enfocar las acciones de control hacia aquellos aspectos estratégicos de la organización.

Al respecto Subía (2013a) concuerda que: 
La necesidad de disponer de informes integrales de evaluación se presenta como una alternativa válida para enfrentar los retos del control externo a las organizaciones. Las orientaciones específicas de las auditorías financieras y de gestión, si bien han proporcionado aportes importantes para el mejoramiento de la gestión institucional, no cubren todos los enfoques. La auditoría integral constituye una oportunidad a los esquemas de control vigentes en el Ecuador. Su adopción en otros países, demuestra la utilidad de un enfoque completo y holístico de la auditoría. (p.5)

La auditoría integral nos ofrece una metodología que permita además de evaluar la información financiera, identificar las diferentes inconformidades de la gestión administrativa en relación, al uso óptimo y de los recursos y al cumplimiento de las metas propuestas, así mismo, garantiza que el control interno de la entidad ofrece la seguridad razonable para el cumplimiento de dichas metas y de la normativa aplicable al ente.

Las revisiones independientes de cada una de las auditorías sobre el cumplimiento de cada uno de sus objetivos particulares, limita al auditor obtener una visión más amplia e importante sobre el logro de las metas misionales.

La experiencia ha demostrado que las acciones de control tradicional, bajo enfoques financieros, de gestión o de cumplimiento, asumidas en forma individual no garantizan acciones de mejoramiento eficaces y más aún un análisis completo de las operaciones administrativas y financieras de las organizaciones en el país. La sinergia que se genera al reunir los propósitos de cada una de las auditorías: financiera, de gestión, de cumplimiento legal y de control interno, nos permitirá obtener resultados favorables a la administración de las mismas (Iza, Ribadeneira y Goyes, 2017, p.4).

De acuerdo a Viloria (2004), la auditoría se ha basado en modelos para su ejercicio que parten de la conjetura de que:

Los sistemas de control interno organizacionales se pueden dividir en dos partes: lo administrativo y lo contable, haciendo énfasis el auditor en lo contable. Ahora bien, la estructura organizacional y su entorno han cambiado, y se exige mayor información al auditor, surgiendo entonces la auditoría integral, entendida ésta como la sumatoria de tipos de auditoria, pero donde prevalece la sectorización organizacional. La visión que se 
propone es que el auditor tome los conceptos del holismo y de la gerencia para realizar eficientemente su trabajo. (p.85)

En relación a lo expuesto, se entiende que una entidad dispone de recursos humanos, materiales y financieros; sin embargo, no se conoce cuál es el nivel de eficiencia, economía, efectividad y transparencia en que son utilizados dichos recursos, porque no se efectúa una verificación o evaluación de estos elementos de la gestión institucional que reporte la situación de los mismos para una adecuada planeación, toma de decisiones y control institucional.

Cuando se dice que no se conoce el nivel de eficiencia, de economía y de efectividad de los recursos, debe entenderse respectivamente que la entidad no evalúa la relación entre los resultados obtenidos y los recursos utilizados; que no toma en cuenta la relación beneficio / costo de los bienes y servicios que utiliza y que no utiliza sus recursos en actividades, funciones, procesos $\mathrm{y}$ procedimientos que no necesariamente facilitan el cumplimiento de metas y objetivos. Esta necesidad de conocer cómo se desarrollan las distintas actividades en la institución, debe ser satisfecha bajo un enfoque global de análisis que cubra todos los frentes de la administración.

En este ámbito, ejecutar una auditoría integral a una institución pública del Cantón Cañar, Provincia Cañar - Ecuador, con el propósito de transformar las acciones de control en verdaderos instrumentos de apoyo que permitan mejorar sus procesos y procedimientos, constituye la esencia de esta investigación, su desarrollo implica la emisión de una opinión sobre la razonabilidad de los saldos expuestos en los estados financieros, el funcionamiento del control interno, el cumplimiento de las principales disposiciones legales y la gestión institucional, por el periodo 2016.

Este objetivo guarda estrecha correspondencia con la hipótesis general que establece: La aplicación de una auditoría integral permitirá alcanzar una evaluación holística de los aspectos financieros, de control interno, de cumplimiento y de gestión, 
que posibilite emitir un informe de aseguramiento orientado a que la dirección de la entidad tome las acciones correctivas necesarias sobre las inconformidades detectadas.

En el presente artículo, en primer lugar, se aborda la exposición de las principales bases teóricas de las diferentes auditorías que conforman la integral; a continuación, se detalla la metodología a seguir para el análisis de cómo se ejecuta la auditoría integral; posteriormente se presentan los resultados, conclusiones $y$, finalmente, se emiten recomendaciones generales que motivan su aplicación.

\section{Marco conceptual}

A fin de alcanzar una mayor comprensión a cerca de la auditoría integral, resulta importante hacer hincapié en las conceptualizaciones que la implican. Al respecto Blanco (2012a) la define como:

El proceso de obtener y evaluar objetivamente, en un periodo determinado, evidencia relativa a la siguiente temática: la información financiera, la estructura de control interno, el cumplimiento de las leyes pertinentes y la conducción ordenada en el logro de las metas y objetivos propuestos; con el propósito de informar sobre el grado de correspondencia entre la temática y los criterios o indicadores establecidos para su evaluación (p.4)

Es integral, puesto que abarca los asuntos relacionados con las otras auditorías, e implica la ejecución de un trabajo con el enfoque por analogía de las revisiones financieras, de cumplimiento, control interno y de gestión, facilitando una visión del conjunto de responsabilidades más importante de la gestión institucional.

De acuerdo con la definición anterior, los objetivos de una auditoría integral ejecutada por un contador público independiente son:

- Determinar si los estados financieros, que se constituyen en objeto de indagación, se presentan de acuerdo con los principios de contabilidad generalmente aceptados y de regulaciones que les son aplicables.

- Establecer si la entidad ha cumplido, en el desarrollo de sus operaciones financieras, administrativas y económicas, con las normas legales que le sean 
aplicables, sus reglamentos, los estatutos y las decisiones de los órganos de dirección y administración.

- Evaluar el sistema global del control interno del ente, con el alcance necesario para dictaminar si funciona efectivamente para la consecución de los siguientes objetivos:

$>$ Efectividad y eficiencia de las operaciones

$>$ Confiabilidad de la información financiera

$>$ Cumplimiento de las leyes y disposiciones aplicables.

- Valorar si la entidad se ha conducido de manera ordenada en el logro de los objetivos previstos y el grado de eficiencia y eficacia con que se han manejado los recursos disponibles.

Revisar el desempeño general de la entidad en relación a sus metas y objetivos es el objetivo primordial de la auditoría integral. Por su amplitud requiere de las siguientes clases de auditoría: financiera, de control interno, de cumplimiento y de gestión.

Desagregando cada una de ellas, destacamos a la auditoría financiera como:

El examen de los registros, comprobantes, documentos y otras evidencias que sustentan los estados financieros de una entidad $\mathrm{u}$ organismo, efectuado por el auditor para formular el dictamen respecto de la razonabilidad con que se presentan los resultados de las operaciones, la situación financiera, los cambios operados en ella y en el patrimonio; para determinar el cumplimiento de las disposiciones legales y para formular comentarios, conclusiones y recomendaciones tendientes a mejorar los procedimientos relativos a la gestión financiera y al control interno (Contraloría General del Estado, 2001, p.11).

Es un examen objetivo, sistemático y profesional, que tiene como objetivo determinar la razonabilidad, integridad y correcta valuación de los saldos expuestos en los estados financieros, expedientes y demás documentos, presentados por la dirección y formular las sugerencias de mejora administrativo - contable.

En otras palabras, la información proporcionada por la auditoría financiera, según lo advierte Subía (2014), es: 
$\checkmark \quad$ Veraz: es decir, que los activos y pasivos sean propiedad de la empresa. Se requieren procedimientos de verificación documental y física para determinar su cumplimiento.

$\checkmark \quad$ Integra: referida a que los registros contables estén completos y que las operaciones sean registradas en el período al que corresponden. Su evaluación se orientará a la revisión de la oportunidad de los sistemas de registro y su correcta clasificación.

$\checkmark$ Correcta valuación: quiere decir que, los activos y pasivos se encuentran revelados de acuerdo a su valor de realización. Se hace necesario, entre otros, cálculos de depreciación, revalorización y registro de cambios en cotizaciones de moneda extranjera. (p.21)

El auditor recaba la evidencia concerniente a estas afirmaciones realizadas por la gerencia y que están contenidas en los estados financieros. Para considerar los distintos tipos de posibles errores que puedan ocurrir, el profesional debe obtener una comprensión de las actividades de control que son relevantes para la auditoría, debe identificar y evaluar los riesgos de error material en función de dichas aseveraciones.

Lo anterior hace notar la necesidad de contar con un adecuado sistema de control interno, que no es otra cosa que, una expresión que se utiliza con el objetivo de detallar las acciones tomadas por los directores, gerentes o administradores de una entidad, para evaluar y monitorear sus actividades.

En aras de mejorar los sistemas de control interno de acuerdo a Ablan y Méndez (2010a), algunos organismos internacionales han propuesto:

modelos que han sido ampliamente aceptados tanto en el sector privado como en el público, entre ellos se encuentra el informe emitido por el Committee of Sponsoring Organizations (COSO) cuyo marco conceptual establece criterios prácticos para la organización y evaluación del sistema de control interno. (p.6)

En este ámbito el Informe COSO se presenta en el entorno organizacional como un marco conceptual de general aceptación, en lo que corresponde al sistema de control interno que deben implementar las organizaciones para valorar la efectividad de sus procedimientos en cuanto a la administración de los riesgos inherentes a sus actividades. 
Mantilla (2005), en su libro Control Interno, recopila información sobre el Informe COSO y define al control interno como:

Un proceso, ejecutado por el consejo de directores, la administración y otro personal de una entidad, diseñado para proporcionar seguridad razonable con miras a la consecución de objetivos en las siguientes categorías: efectividad y eficiencia en las operaciones, confiabilidad de la información financiera y cumplimiento de las leyes y regulaciones aplicables. (p.14)

Se entiende entonces que, el control interno no es competencia de determinada oficina o grupo de personas, sino que, por el contrario, nace en la actitud de las personas y se manifiesta a través de las diferentes actuaciones de los seres humanos, en los distintos escenarios o espacios que ocupan, tanto en el contexto familiar, social o laboral. Comprende el plan de la organización y todos los métodos coordinados y medidas adoptadas dentro de una empresa con el fin de salvaguardar sus activos.

Si se logra identificar perfectamente cada uno de estos objetivos, se puede afirmar que se conoce el significado de control interno. En este sentido sus objetivos son los siguientes:

$\checkmark$ Información financiera veraz, referida con la elaboración de los estados financieros confiables, la contabilidad implica, operaciones, procesos y produce información financiera, para que los usuarios tomen decisiones, será fidedigna si la entidad cuenta con un sistema que permita su estabilidad, objetividad y verificabilidad. Si se cuenta con un apropiado sistema de información financiera se ofrecerá mayor protección a los recursos de la empresa a fin de evitar sustracciones y demás peligros que puedan amenazarlos.

$\checkmark$ Efectividad y eficiencia de las operaciones, relativa a la seguridad de que las actividades se cumplan cabalmente con un mínimo de esfuerzo y utilización de recursos y un máximo de utilidad de acuerdo con las autorizaciones generales especificadas por la administración.

$\checkmark$ Cumplimiento del marco normativo, en relación a que toda acción que se emprenda por parte de la dirección de la organización, debe estar enmarcada dentro las 
disposiciones legales del país y debe obedecer al cumplimiento de toda la normatividad que le sea aplicable al ente, incluyendo las políticas emitidas por la alta dirección.

De acuerdo a Navarro y Ramos (2016), el informe COSO constituye un:

Modelo de evaluacion de las normas, procedimientos y politicas llevadas a cabo por la junta directiva, la gerencia y demas integrantes de la organizacion, con la finalidad de brindar seguridad razonable con respecto al logro de los objetivos institucionales relacionados con el nivel de eficiencia y efectividad con que se ejecutan las operaciones y actividades corporativas, el grado de fiabilidad que exhibe la informacion financiera generada por los sistemas de informacion y el nivel de cumplimiento por parte de la empresa de las disposiciones normativas y estatutarias, se han establecido cinco componentes basicos que se encuentran interrelacionados unos con otros. (p.254)

En resumen el informe $\mathrm{COSO}$ es un proceso integral y dinámico que permite analizar los componentes referidos al ambiente de control, evaluación de riesgos, actividades de control, información y comunicación y supervisión o monitoreo de las responsabilidades que cada funcionario asume en sus puestos de trabajo.

La auditoría del control interno evalúa si el sistema de control interno de la entidad está funcionando adecuadamente para el cumplimiento de los objetivos descritos anteriormente. Las afirmaciones constan en su naturaleza, por lo tanto verifica el diseño, aplicación y actualización del sistema de control interno de la organización.

Para su aplicación el Informe Coso incluye tres métodos de evaluación: el cuestionario, narrativo o descriptivo y el método gráfico o diagramas de flujo, según Estupiñan (2006), el primer método consiste en:

Diseñar cuestionarios con base en preguntas que deben ser contestadas por los funcionarios y personal responsable de las distintas áreas de la empresa bajo exámen. Las preguntas son formuladas de tal forma que la respuesta afirmativa indique un punto óptimo en el sistema de control interno y una respuesta negativa indique una debilidad o un aspecto no muy confiable. (p.160). 
Las preguntas estan orientadas a medir la efectividad del control interno en cada uno de los siguientes componentes: ambiente de control, evaluación de los riesgos, actividades de control, información y comunicación y supervisión o monitoreo.

El método narrativo, describe las características y procesos relevantes del sistema de control interno de la entidad, clasificado por departamentos, funcionarios y actividades que intervienen en dicho sistema.

En el método gráfico, el profesional detalla por medio de símbolos los procedimientos que se ejecutan en cada uno de los distintos departamentos, con el fin de proporcionar una idea clara y completa de la forma como se ejecutan las actividades.

Por otro lado la auditoria de cumplimiento, de acuerdo a Blanco (2012b) es:

La comprobación o examen de las operaciones financieras, administrativas, económicas y de otra índole de una entidad para establecer que se han realizado conforme a las normas legales, reglamentarias, estatutarias y de procedimientos que le son aplicables.

Esta auditoría se practica mediante la revisión de los documentos que soportan legal, técnica, financiera y contablemente las operaciones para determinar si los procedimientos utilizados y las medidas de control interno están de acuerdo con las normas que le son aplicables y si dichos procedimientos están operando de manera efectiva y son adecuados para el logro de los objetivos de la entidad. (p.362)

Entonces la evaluación, se practica con el objetivo de comprobar que la entidad o una de sus dependencias procedió de acuerdo a los preceptos legales que la rigen, a las normas que los órganos administrativos competentes le han fijado, a los principios de contabilidad generalmente aceptados en lo pertinente a su información financiera y a las reglas que internamente se hayan impuesto para ordenar sus actividades.

El incumplimiento de leyes y regulaciones a las que se encuentran sujetas las organizaciones puede poner en riesgo su continuidad o generar grandes pérdidas financieras por penalidades y recargos que pueden afectar la imagen, credibilidad y hasta cerrar su negocio u organización.

Para evitar tal incumplimiento, es aconsejable, que en la entidad se establezca procedimientos para vigilar el cumplimiento legal en la organización, se instituya un 
ambiente de honestidad; se elabore códigos y manuales de conducta a seguir por el personal, entre otras acciones.

Entre las políticas y procedimientos que pueden ayudar a la administración para controlar sus responsabilidades en la prevención y detección de incumplimientos, Espinoza (2013), recomienda las siguientes:

$\checkmark$ Monitorear los requisitos legales y asegurar que los procedimientos de operación estén diseñados para cumplir con estos requisitos.

$\checkmark$ Instituir y operar sistemas apropiados de control interno.

$\checkmark$ Desarrollar, hacer público, y seguir un código de conducta.

$\checkmark$ Asegurar que los empleados están apropiadamente entrenados y comprenden el Código de Ética.

$\checkmark$ Monitorear el cumplimiento del Código de Ética y actuar apropiadamente para disciplinar a los empleados que dejan de cumplir con él,

$\checkmark$ Contratar asesores legales parar ayudar en el monitoreo de los requisitos legales.

$\checkmark$ Mantener un registro de leyes importantes con las que la entidad tiene que cumplir dentro de su industria particular y un registro de quejas. (p.27)

En el caso de la auditoría de cumplimiento, los criterios no pueden ser normalizados, dependen de las actividades y el objeto social de la empresa. Entre las principales disposiciones legales a las que se encuentra sujeta la institución objeto de estudio se destacan las siguientes:

- Constitución Política de la República del Ecuador (Asamblea Nacional Constituyente, 2008).

- Ley del Deporte, Educación Física y Recreación y su reglamento de aplicación (Asamblea Nacional Constituyente, 2010a).

- Reglamento de la Ley Orgánica de la Contraloría General (Presidencia de la República, 2003).

- Reglamento para aplicación Ley de Régimen Tributario Interno, LORTI (Presidencia de la República, 2010a).

- Ley Orgánica del Sistema Nacional de Contratación Pública (Presidencia de la República, 2008a). 
- Reglamento General a la Ley Orgánica del Servicio Público (Presidencia de la República, 2011a).

- Demás disposiciones legales que norman el funcionamiento de las entidades deportivas.

La normativa tributaria, laboral, cambiaria, entre otras constituye también, elemento clave de la evaluación. En el sector público a diferencia del sector privado, debe realizarse únicamente lo que está permitido por la ley, por consiguiente, el derecho público es el referente en el control del cumplimiento.

Finalmente, la necesidad de reajustar la gestión o la organización de la misma, es uno de los principales motivos por el cual un ente puede decidir emprender una auditoría de gestión, que ayude a analizar, diagnosticar y establecer recomendaciones, con el fin de conseguir con éxito una estrategia.

Surge entonces la auditoría de gestión, de la necesidad de medir y cuantificar los logros alcanzados en un período de tiempo determinado. Emerge como una manera efectiva de poner en orden los recursos de la entidad para lograr un mejor desempeño y productividad.

Franklin (2007), define a la auditoría de gestión como "la revisión analítica total o parcial de una organización con el propósito de precisar su nivel de desempeño y perfilar oportunidades de mejora para innovar valor y lograr una ventaja competitiva sustentable" (p.11). Actualmente para ser reconocida como una institución eficiente y competitiva, se requieren los más altos estándares de calidad, por lo cual las organizaciones han optado por aplicar evaluaciones de su gestión, para determinar la conformidad de los procesos.

En esta línea, Velásquez (2013), sostiene que la auditoría de gestión es:

Una actividad que sobre la base de un modelo y un marco normativo, permite determinar el grado de eficiencia, eficacia y calidad en la utilización de los recursos disponibles, el desarrollo de procesos y la entrega de productos o de servicios, acordes con las necesidades de los clientes, los mismos que podrán ser mejorados en forma continua, a través de la aplicación de las recomendaciones emanadas del informe de auditoría 
(p.14).

Tomando en consideración que en toda organización se procesa información financiera, así como administrativa y operacional, como consecuencia de sus actividades y que tienen incidencia en el logro de sus objetivos, metas y programas, es importante valorarlas a través de indicadores, para establecer el grado de eficiencia, eficacia, economía, efectividad, entre otros, con el cual fueron alcanzados.

Para concluir con la conceptualización de la auditoría de gestión, la Contraloría General del Estado (2011), la define como:

El examen sistemático y profesional, efectuado por un equipo multidisciplinario, con el propósito de evaluar la eficacia de la gestión de una entidad, programa, proyecto u operación, en relación a sus objetivos y metas; de determinar el grado de economía y eficiencia en el uso de los recursos disponibles; y, de medir la calidad de los servicios, obras o bienes ofrecidos, y el impacto socio-económico derivado de sus actividades. (p.6)

Por su enfoque, involucra una revisión sistemática de las actividades de una organización en correspondencia a determinados objetivos y metas, y respecto a la utilización eficiente y económica de los recursos.

Entre los motivos que dan lugar a una auditoría de gestión está la necesidad de controlar la gestión de la empresa en sus diferentes niveles. En este caso, se persigue el objetivo de establecer un control de eficacia, eficiencia y calidad y su insumo básico lo constituyen los indicadores de gestión, orientados no sólo a cifras, sino a resultados y procesos. Ello conduce a precisar con suficiente claridad a qué nos referimos cuando hablamos de indicador de gestión. Adoptamos entonces el planteamiento esclarecedor que hace Subía (2013b), sobre el término:

Indicador es la medición de un objetivo, meta o proceso. Deben identificar claramente la variable a medir. Los indicadores para medir eficiencia, eficacia y calidad son claramente diferenciables. No necesariamente debe presentarse en porcentaje, un indicador es aplicable si se muestra en cantidades o valores absolutos, sin embargo, en su análisis es necesario mantener consistencia con la unidad de medida y el estándar (p.16-17) 
En efecto los indicadores de gestión constituyen los "signos vitales" de una organización. De ahí la importancia de contar con un mínimo de indicadores que garanticen disponer de información constante, real y precisa sobre aspectos tales como la efectividad, la eficacia, la eficiencia, la productividad, la calidad, la ejecución presupuestal, la incidencia de la gestión.

Para lograr una gestión eficaz y eficiente es importante diseñar un sistema de control de gestión que apoye a la administración y le permita evaluar a la entidad, facilitando a cada uno de sus usuarios, información oportuna y efectiva sobre el comportamiento de las variables críticas para el éxito, a través de los indicadores de gestión que hayan sido previamente definidos.

Para concluir, es importante destacar que la auditoría integral constituye un sistema novedoso en el proceso de gestión eficiente de toda organización, por lo que requiere ser conceptualizada desde la articulación de los procesos que integran los otros enfoques de auditoría: por analogía de las revisiones financieras, de cumplimiento, control interno y de gestión, a fin de facilitar la construcción de una visión holística del conjunto de responsabilidades que atañen a la gestión institucional. En este sentido, coincidimos con los planteamientos de Subía (2013a), quien destaca la visión innovadora que se alcanza al integrar los objetivos y propósitos de las auditorías para obtener una evaluación global de los resultados y del desempeño de la organización.

\section{Materiales y métodos}

La metodología para llevar a cabo este estudio, cuyo objetivo se orienta a analizar la ejecución de la auditoría integral en una institución pública, es fundamentalmente cualitativo. Busca corroborar la hipótesis general de trabajo que plantea: La aplicación de una auditoría integral permitirá alcanzar una evaluación holística de los aspectos financieros, de control interno, de cumplimiento y de gestión, que permita emitir un informe de aseguramiento orientado a que la dirección de la entidad tome las acciones correctivas necesarias sobre las inconformidades detectadas. 
En este contexto, para este estudio el procedimiento a seguir consistió en ejecutar el examen en forma ordenada y con una secuencia congruente de actividades, las mismas que iniciaron con una revisión documental de las actividades principales de la institución, para ubicar exactamente la extensión del trabajo a desarrollar, se revisó informes de auditorías anteriores, normativa y disposiciones legales aplicables, información financiera, planes estratégicos, operativos, entre otros.

Su desarrollo implicó acumular y evaluar evidencia con la finalidad de descubrir debilidades o vulnerabilidades en la estructura de la organización. El estudio realizado exigió la utilización de requerimientos de:

Investigación documental: para sustentar el estudio se ha recurrido a la selección y recopilación de información proveniente de documentos oficiales tales como: estados financieros, para determinar la razonabilidad de los saldos expuestos; base legal, orientada a establecer el grado de cumplimiento de la normativa aplicable; el plan operativo anual, con la finalidad de verificar el cumplimiento de las metas fijadas así como el uso de los recursos planificados para su consecución; expedientes del talento humano, planillas de aportes a la seguridad social, roles de pago, manual de funciones y estatutos de la institución donde se ejecutó la auditoría correspondientes al año 2016 y que servirá de evidencia para fundamentar el informe.

El trabajo fue intencional y se desarrolló bajo los criterios de cada una de las auditorías que conforman la auditoría integral, es decir, para la auditoría financiera se aplicaron las Normas Internacionales de Contabilidad (NIC) (Normas Internacionales de Contabilidad No.1, 2006a).

Para la auditoría del control interno que tiene como objetivo fundamental evaluar el sistema de control interno. El informe COSO de acuerdo a lo expuesto en párrafos anteriores por Ablan y Méndez (2010b), constituye un modelo de evaluación del control interno aplicable para empresas privadas y públicas.

El cuestionario de control interno se aplicó al Presidente, Secretario, Tesorero y Jefe de Talento Humano, debido a que son los responsables de los diferentes procesos 
que se desarrollan en la institución. Las preguntas fueron distribuidas de acuerdo a los componentes del control interno y considerando las funciones que cada uno desempeñan.

La auditoría de cumplimiento demandó la evaluación del grado de cumplimiento de las principales disposiciones legales a las que se encuentra sujeta la institución como:

- Ley del Deporte, Educación Física y Recreación y su reglamento de aplicación (Asamblea Nacional Constituyente, 2010b).

- Reglamento para aplicación Ley de Régimen Tributario Interno, LORTI (Presidencia de la República, 2010b).

- Ley Orgánica del Sistema Nacional de Contratación Pública (Presidencia de la República, 2008b).

- Reglamento General a la Ley Orgánica del Servicio Público (Presidencia de la República, 2011b).

En lo que respecta a la auditoría de gestión, se orienta a la evaluación de la eficiencia, eficacia y calidad de los productos y servicios proporcionados por una organización. Bajo este enfoque es necesario señalar en forma clara, la conceptualización de los siguientes criterios: La eficiencia se relaciona con la optimización en el uso de los recursos y la eficacia mide el logro de las metas y objetivos institucionales.

\section{Resultados}

Con base a los procedimientos de trabajo y con la evidencia obtenida se expone los siguientes resultados, por cada una de las auditorías que conforman la auditoría integral:

\section{Auditoría Financiera}

Luego de analizar cada uno de los rubros que conforman los estados 
financieros de la entidad, se determinó que: el valor correspondiente al Aporte Personal por el año 2016, ha sido consignado en la cuenta Aporte Patronal, por lo tanto, su saldo no se presenta razonablemente, en contradicción con la Norma NIC 1 Presentación de Estados Financieros, que manifiesta que:

Los estados financieros reflejarán fielmente, la situación, el rendimiento financiero y los flujos de efectivo de la entidad. La imagen fiel exige la representación fiel de los efectos de las transacciones, así como de otros eventos y condiciones, de acuerdo con las definiciones y los criterios de reconocimiento de activos, pasivos, ingresos y gastos fijados en el Marco Conceptual. Se presumirá que la aplicación de las NIIF, acompañada de informaciones adicionales cuando sea preciso, dará lugar a estados financieros que proporcionen una presentación razonable (Normas Internacionales de Contabilidad No.1, 2006b, p.4).

\section{Auditoría de Control Interno}

Para el examen se aplicó el cuestionario de control interno, el mismo que tiene como finalidad medir la efectividad en cada uno de los siguientes componentes: ambiente de control, evaluación de los riesgos, actividades de control, información y comunicación y monitoreo, así como determinar el nivel de confianza y riesgo de control. Para obtener el nivel de confianza se divide calificación total (CT) para el total de preguntas o ponderación total (PT), que en comparación con el 100\% representaria el riesgo de control.

$\mathrm{Al}$ respecto se establece los siguientes resultados:

Tabla 1

Resultados de la aplicación de la auditoría de control interno

\begin{tabular}{lc|ccccc}
\multicolumn{2}{c}{ Componentes } & $\begin{array}{c}\text { Total } \\
\text { preguntas }\end{array}$ & Calificación & $\begin{array}{c}\text { \% Nivel de } \\
\text { confianza }\end{array}$ & $\begin{array}{c}\text { \% Nivel de } \\
\text { riesgo }\end{array}$ & $\begin{array}{c}\text { Nivel de } \\
\text { riesgo }\end{array}$ \\
\hline $\begin{array}{l}\text { Ambiente } \\
\begin{array}{l}\text { Control } \\
\text { Evaluación } \\
\text { riesgo }\end{array}\end{array}$ & 20 & 14 & $70 \%$ & $30 \%$ & Moderado \\
$\begin{array}{l}\text { Actividades } \\
\text { Control }\end{array}$ & 8 & 80 & 1 & $13 \%$ & $88 \%$ & Alto \\
\end{tabular}




\begin{tabular}{r|ccccl}
$\begin{array}{l}\text { Información } \\
\begin{array}{l}\text { Comunicación } \\
\text { Monitoreo }\end{array}\end{array}$ & 2 & 2 & $100 \%$ & $0 \%$ & Bajo \\
\multicolumn{1}{c}{ SUMAS } & $\mathbf{1 1 2}$ & $\mathbf{7 1}$ & $63 \%$ & $37 \%$ & Moderado
\end{tabular}

Fuente: Elaboración propia.

\section{Tabla 2}

Nivel de confianza y riego del sistema de control interno

Tabla del nivel de confianza

\begin{tabular}{ll|lr}
\hline 76 al $95 \%$ & $=$ alto & Calificación total & $\mathbf{7 1}$ \\
51 al $75 \%$ & $=$ moderado & Ponderación total & $\mathbf{1 1 2}$ \\
15 al $50 \%$ & $=$ bajo & Nivel de confianza & $\mathbf{6 3 \%}$ \\
& & Nivel confianza - N/C & Moderada \\
& Nivel de riesgo $-\mathrm{N} / \mathrm{R}$ & Moderado
\end{tabular}

Fuente: Elaboración propia.

Las inconformidades encontradas a través de la aplicación del cuestionario representan aquellos aspectos o procedimientos que la institución no dispone o no aplica de manera adecuada, entre ellos se encontró los siguientes:

La institución no dispone de una planificación estratégica, que permita programar sus actividades, para conseguir los objetivos a plazo fijo, incumpliendo la Norma de Control Interno 200-02 Administración Estratégica, que establece que:

Las entidades del sector público y las personas jurídicas de derecho privado que dispongan de recursos públicos implantarán, pondrán en funcionamiento y actualizarán el sistema de planificación, así como el establecimiento de indicadores de gestión que permitan evaluar el cumplimiento de los fines, objetivos y la eficiencia de la gestión institucional (Contraloría General del Estado, 2009, p.5).

No dispone de políticas y prácticas de talento humano que fomente un ambiente ético de profesionalismo y transparencia en sus prácticas diarias, en oposición a la Norma de Control Interno, 200-03 Políticas y Prácticas de Talento Humano, que 
determina que: "el control interno incluirá las políticas y prácticas necesarias para asegurar una apropiada planificación y administración del talento humano de la institución, de manera que se garantice el desarrollo profesional y asegure la transparencia, eficacia y vocación de servicio" (Contraloría General del Estado, 2009, p.6).

Asigna a un solo servidor para que controle o tenga a su cargo todas las etapas claves de un proceso u operación, infringiendo la Norma de Control Interno 401-01 Separación de funciones y rotación de labores, que establece que:

La máxima autoridad y los directivos de cada entidad tendrán cuidado al definir las funciones de sus servidoras y servidores y de procurar la rotación de las tareas, de manera que exista independencia, separación de funciones incompatibles y reducción del riesgo de errores o acciones irregulares (Contraloría General del Estado, 2009, p.10).

No se han implantado procedimientos de control interno que aseguren el cumplimiento de las fases del ciclo presupuestario. En oposición con la Norma de Control Interno 402-01 Responsabilidad de control, que establece que:

La máxima autoridad de una entidad, u organismo del sector público, dispondrá a los responsables de las unidades inherentes a la materia, el diseño de los controles que se aplicarán para asegurar el cumplimiento de las fases del ciclo presupuestario en base de las disposiciones legales, reglamentarias y políticas gubernamentales, sectoriales e institucionales, que regulan las actividades del presupuesto y alcanzar los resultados previstos (Contraloría General del Estado, 2009, p.11).

No aplica los principios y normas de contabilidad gubernamental, para el registro de las operaciones y presentación de la información financiera. Incumpliendo con la Norma de Control Interno 405-01 Aplicación de los principios y normas técnicas de contabilidad gubernamental, que manifiesta que: "la contabilidad gubernamental se basará en principios y normas técnicas emitidas sobre la materia, para el registro de las operaciones y la preparación y presentación de información financiera, para la toma de decisiones" (Contraloría General del Estado, 2009, p.24). 
No cuenta con un reglamentado sobre el uso, custodia y conservación de los bienes de larga duración entregados a los servidores para el desempeño de sus funciones, incumpliendo con la Norma de Control Interno 406-08 Uso de bienes de larga duración, que establece que: “en cada entidad pública los bienes de larga duración se utilizarán únicamente en las labores institucionales y por ningún motivo para fines personales, políticos, electorales, religiosos u otras actividades particulares" (Contraloría General del Estado, 2009, p.34).

La Secretaría de la institución, no dispone de expedientes individuales que contengan la documentación general, laboral, información sobre el ingreso, capacitación, evaluaciones, ascensos, promociones y retiro de cada uno de los servidores, incumpliendo así la Norma de Control Interno 407-10 Información actualizada del personal, que determina que: "la unidad de administración de talento humano será responsable del control de los expedientes de las servidoras y servidores de la entidad, de su clasificación y actualización" (Contraloría General del Estado, 2009, p.40).

\section{Auditoría de cumplimiento}

De toda la base normativa descrita anteriormente a continuación se detalla las normas o reglamentos que la que la institución no cumple a cabalidad.

En este ámbito al revisar los expedientes de los empleados de la entidad, se pudo evidenciar, que los mismos no han presentado la certificación de no tener impedimento legal para ingresar al sector público emitida por el Ministerio de Relaciones Laborales, esta normativa establece que:

Para ocupar un puesto en el servicio público, debe cumplirse con los requisitos establecidos en el artículo 5 de la LOSEP, a cuyo efecto las personas deben cumplir con lo siguiente:

1.- Presentar la certificación de no tener impedimento legal para ingresar al servicio público emitida por el Ministerio de Relaciones Laborales (Presidencia de la República, 2011c, art. 3). 
Así mismo se determinó que no realiza las adquisiciones mediante el portal de Compras Públicas, incumpliendo así el marco regulatorio que establece que:

Las compañías mercantiles cualquiera hubiere sido o fuere su origen, creación o constitución que posean o administren bienes, fondos, títulos, acciones, participaciones, activos, rentas, utilidades, excedentes, subvenciones y todos los derechos que pertenecen al Estado y a sus instituciones, sea cual fuere la fuente de la que procedan, inclusive los provenientes de préstamos, donaciones y entregas que, a cualquier otro título se realicen a favor del Estado o de sus instituciones; siempre que su capital, patrimonio o los recursos que se le asignen, esté integrado en el cincuenta $(50 \%)$ por ciento o más con participación estatal; y en general toda contratación en que se utilice, en cada caso, recursos públicos en más del cincuenta (50\%) por ciento del costo del respectivo contrato. Se exceptúan las personas jurídicas a las que se refiere el numeral 8 del artículo 2 de esta Ley, que se someterán al régimen establecido en esa norma (Presidencia de la República, 2008c, art. 1).

\section{Auditoría de Gestión}

Mediante el análisis de indicadores, efectuado al cumplimiento de las metas establecidas en la Planificación Operativa Anual, correspondiente al año 2016 de la institución, se determina el siguiente grado de eficacia:

\section{Tabla 3}

\section{Resultados de la aplicación de la auditoría de gestión para determinar el grado de eficacia en el cumplimiento de metas}

\section{Nombre del Indicador}

Número de deportistas participantes

Porcentaje de reducción del nivel de sedentarismo

Porcentaje de deportistas de atletismo clasificados a los Juegos Cantonales y Nacionales

Número de deportistas con capacidades especiales participantes

Porcentaje de deportistas clasificados a los Juegos Cantonales

\begin{tabular}{|c|c|c|c|}
\hline Estándar & Ejecutado & Cálculo & Brecha \\
\hline & & $\begin{array}{c}\text { Ejecutado } \\
\text { Estándar }\end{array}$ & \\
\hline 2225 & 2184 & $98 \%$ & $-2 \%$ \\
\hline $10 \%$ & $8 \%$ & $80 \%$ & $-20 \%$ \\
\hline $33 \%$ & $33 \%$ & $100 \%$ & $0 \%$ \\
\hline 110 & 110 & $100 \%$ & $0 \%$ \\
\hline $16 \%$ & $16 \%$ & $100 \%$ & $0 \%$ \\
\hline
\end{tabular}


Porcentaje de deportistas clasificados a Competencias Internacionales

Porcentaje de deportistas clasificados a Seleccionados Provinciales

Porcentaje de deportistas clasificados a Campeonatos Nacionales

Porcentaje de deportistas con el mejor desempeño en la disciplina de Karate Do Número de deportistas capacitados

Promedio de metas cumplidas Fuente: Elaboración propia

\begin{tabular}{|cccc}
$13 \%$ & $7 \%$ & $55 \%$ & $-45 \%$ \\
$60 \%$ & $60 \%$ & $100 \%$ & $0 \%$ \\
$10 \%$ & $7 \%$ & $70 \%$ & $-30 \%$ \\
$12 \%$ & $7 \%$ & $58 \%$ & $-42 \%$ \\
65 & 55 & $85 \%$ & $-15 \%$ \\
& & $\mathbf{8 5 \%}$ & $\mathbf{- 1 5 \%}$
\end{tabular}

Con respecto al nivel de eficiencia, mediante el análisis de los rubros identificados por tipo de gasto, establecidos en el Plan Operativo Anual, correspondiente al año 2016 se determina un nivel de asignación de recursos a las diferentes actividades del $98.55 \%$, conforme se puede visualizar en la tabla 4 .

\section{Tabla 4}

\section{Resultados de la aplicación de la auditoría de gestión para determinar el grado} de asignación de los recursos a las actividades. Nombre del Indicador (TIPO DE GASTO)

Gasto corriente directo

Gasto corriente indirecto

Gasto corriente mantenimiento

Gasto de Inversión

Total

\begin{tabular}{|rrrr} 
Planificado & Ejecutado & \multicolumn{1}{c}{ Cálculo } & Brecha \\
\cline { 3 - 3 } & & \multicolumn{1}{c}{$\begin{array}{c}\text { Ejecutado / } \\
\text { Planificado }\end{array}$} & \\
& & $95.85 \%$ & $-4.15 \%$ \\
\hline 20402.80 & 29141.64 & $99.04 \%$ & $-0.96 \%$ \\
23887.91 & 23659.24 & $99.87 \%$ & $-0.13 \%$ \\
10858.14 & 10843.91 & $99.43 \%$ & $-0.57 \%$ \\
43432.58 & 43183.72 & $\mathbf{9 8 . 5 5 \%}$ & $\mathbf{- 1 . 4 5 \%}$ \\
$\mathbf{1 0 8 5 8 1 . 4 3}$ & $\mathbf{1 0 6 8 2 8 . 5}$ & &
\end{tabular}

Fuente: Elaboración propia

\section{Conclusiones}

La Auditoría Integral permitió evaluar con un enfoque de sistemas, el grado y forma de cumplimiento de los objetivos de la institución, de la relación con su entorno, así como de sus operaciones, y para poder verificar los puntos débiles de la organización con el fin de tomar medidas y precauciones a tiempo. 
En su desarrollo se identificó todas las actividades, sistemas y controles importantes de la entidad, clasificando aquellos que son esenciales para la administración y el uso adecuado de los recursos.

Así mismo, se consideraron componentes importantes de la institución, que sirven de base para el cumplimiento de sus objetivos misionales, y que, a través del informe del examen especial de auditoría integral, los directivos dispongan de una herramienta, que les permita mejorar sus procesos.

Para la realización de la auditoría de control interno se utilizó cuestionarios de control interno basados en el informe COSO, obteniendo un nivel de confianza moderada y consecuentemente un nivel de riesgo moderado.

Los estados financieros de la entidad se presentan razonablemente, a pesar de no disponer de un sistema de contabilidad gubernamental, que brinde la posibilidad de proporcionar información financiera confiable y oportuna.

Con respecto a la evaluación de la normativa y legislación aplicable, podemos mencionar entre las más importantes, el incumplimiento de las siguientes leyes:

- Ley Orgánica del Sistema Nacional de Contratación Pública (Presidencia de la República, 2008c).

- Reglamento General a la Ley Orgánica del Servicio Público (Presidencia de la República, 2011c).

En lo que se refiere a la auditoría de gestión se evaluó la eficacia y eficiencia en la ejecución de la planificación operativa anual (POA), en donde se pudo evidenciar que la institución, no cumple con la ejecución mensual planificada y no presenta las respectivas reprogramaciones, con la finalidad de contar con una correcta ejecución a lo planificado. Adicionalmente, no cumple con la ejecución y monto presupuestado según los meses de devengamiento planteados en el POA.

Mediante la aplicación de los indicadores de eficacia y eficiencia establecidos en la planificación operativa anual de la institución se evidenció: 
- La entidad cumple en un $85 \%$ las actividades programadas

- Cumple en un $98.55 \%$ el uso de los recursos a nivel general.

\section{Recomendaciones}

Considerando a la auditoría Integral como el proceso de obtener y evaluar objetivamente, en un período determinado, evidencia relativa a la información financiera, al comportamiento económico y al manejo de una entidad. Se plantea las siguientes recomendaciones:

Aplicarla como herramienta de control completa de la gestión de las instituciones tanto públicas como privadas; para que emita una opinión sobre la calidad, eficiencia, eficacia y economía de las operaciones; razonabilidad de la información financiera y económica; cumplimiento de las normas legales, administrativas, contables y financieras; asimismo para que pueda prevenir y detectar errores o irregularidades, también para que proponga las mejoras, correcciones y soluciones y finalmente realice el seguimiento y la asesoría que necesitan estas entidades para optimizar su gestión institucional.

No solamente utilizar el proceso normal de la auditoría integral (planeación, ejecución e informe), sino que deben explotar el valor agregado que facilita este tipo de auditoría (supervisión o monitoreo de recomendaciones facilitadas, asesoría y consultoría en control y gestión institucional). De este modo la auditoría integral será la respuesta efectiva para la prevención, detección y solución de los riesgos, que pueden afectar a la organización.

Para prevenir, detectar y enfrentar el riesgo es importante que las organizaciones apliquen un conjunto de actividades de control como autorizaciones, verificaciones, conciliaciones, análisis de operaciones, salvaguarda de activos, segregación de funciones y otras; las mismas que deben ser parte del control interno institucional. La auditoría integral examina el cumplimiento de estas acciones. 
Se recomienda implementar diversas actividades de control, porque las mismas contribuyen al uso racional y efectivo de los recursos y facilitan alcanzar los objetivos establecidos en las normas.

Para efectos de optimizar la gestión integral institucional, es necesario aplicar la eficiencia, eficacia y economía en la utilización de los recursos; estos elementos facilitarán el mejoramiento continuo y por ende la optimización. La auditoría integral facilita esta labor.

\section{Referencias bibliográficas}

Ablan, Nayibe y Méndez, Zayda (2010a). Los sistemas de control interno en los entes descentralizados estatales y municipales desde la perspectiva COSO. Revista Visión Gerencial. No. 1. Venezuela (Pp. 5-17).

Ablan, Nayibe y Méndez, Zayda (2010b). Los sistemas de control interno en los entes descentralizados estatales y municipales desde la perspectiva COSO. Revista Visión Gerencial. No. 1. Venezuela (Pp. 5-17).

Asamblea Nacional Constituyente. (2008). Constitución Política de la República del Ecuador, Registro Oficial No. 449, de 20 de Octubre. Ecuador.

Asamblea Nacional Constituyente. (2010a). Ley del Deporte, Educación Física y Recreación, Registro Oficial No. 255, de 11 de Agosto. Ecuador.

Asamblea Nacional Constituyente. (2010b). Ley del Deporte, Educación Física y Recreación, Registro Oficial No. 255, de 11 de Agosto. Ecuador.

Blanco, Yanel (2012a). Auditoría integral: normas y procedimientos (segunda edición). Ecoe Ediciones. Colombia.

Blanco, Yanel (2012b). Auditoría integral: normas y procedimientos (segunda edición). Ecoe Ediciones. Colombia.

Contraloría General del Estado. (2001). Manual de Auditoría Financiera, Acuerdo No. 016 CG, Agosto. Ecuador.

Contraloría General del Estado. (2011). Guía Metodológica para la Auditoría de Gestión, Acuerdo No. 047 CG, de 22 de Diciembre. Ecuador.

Contraloría General del Estado. (2009). Normas de Control Interno de la Contraloría General del Estado, Acuerdo No.39 CG, de 14 de Diciembre. Ecuador.

Espinoza, María (2013). Auditoría de cumplimiento I (primera edición). Editorial Ediloja. Ecuador.

Estupiñan, Rodrigo (2006). Control interno y fraudes (segunda edición). Ecoe Ediciones. Colombia. 
Franklin, Enrique (2007). Auditoría Administrativa: gestión estratégica del cambio (segunda edición). Editorial Pearson Educación. México.

Iza, Karina; Ribadeneira, Carlos y Goyes, Jorge (2017). Examen de auditoría integral al área de crédito de la Cooperativa de Ahorro y Crédito San José Ltda., correspondiente al período 2013. Revista Observatorio de la Economía Latinoamericana. julio 2017. Ecuador (Pp 1-15). En línea: http://www.eumed.net/cursecon/ecolat/ec/2017/cooperativa-san-jose.html consulta: 18/02/2019.

Mantilla, Samuel (2005). Control Interno: Informe COSO (cuarta edición). Ecoe Ediciones. Colombia.

Navarro, Franlin y Ramos, Liliana (2016). El control interno en los procesos de producción de la industria litográfica en Barranquilla. Revista Equidad y Desarrollo. No. 25. Colombia (Pp. 245-267).

Normas Internacionales de Contabilidad No.1 (2006a). Presentación de Estados Financieros. Extraído de

http://www.normasinternacionalesdecontabilidad.es/nic/pdf/nic01.pdf Consulta: 18/02/2019.

Normas Internacionales de Contabilidad No.1 (2006b). Presentación de Estados Financieros. Extraído de

http://www.normasinternacionalesdecontabilidad.es/nic/pdf/nic01.pdf Consulta: 18/02/2019.

Presidencia de la República. (2003). Reglamento de la Ley Orgánica de la Contraloría General, Registro Oficial No. 119, de 07 de Julio. Ecuador.

Presidencia de la República. (2010a). Reglamento para aplicación Ley de Régimen Tributario Interno, LORTI, Decreto Ejecutivo No. 374, de 08 de Junio. Ecuador.

Presidencia de la República. (2010b). Reglamento para aplicación Ley de Régimen Tributario Interno, LORTI, Decreto Ejecutivo No. 374, de 08 de Junio. Ecuador.

Presidencia de la República. (2011a). Reglamento General a la Ley Orgánica del Servicio Público, Registro Oficial Suplemento 418, de 01 de Abril. Ecuador. Presidencia de la República. (2011b). Reglamento General a la Ley Orgánica del Servicio Público, Registro Oficial Suplemento 418, de 01 de Abril. Ecuador. Presidencia de la República. (2011c). Reglamento General a la Ley Orgánica del Servicio Público, Registro Oficial Suplemento 418, de 01 de Abril. Ecuador. 
Presidencia de la República. (2008a). Ley Orgánica del Sistema Nacional de Contratación Pública, Registro Oficial Suplemento 395, de 04 de Agosto. Ecuador.

Presidencia de la República. (2008b). Ley Orgánica del Sistema Nacional de Contratación Pública, Registro Oficial Suplemento 395, de 04 de Agosto. Ecuador.

Presidencia de la República. (2008c). Ley Orgánica del Sistema Nacional de Contratación Pública, Registro Oficial Suplemento 395, de 04 de Agosto. Ecuador.

Subía, Jaime (2014). Informe de Auditoría Integral (primera edición). Editorial Ediloja. Ecuador.

Subía, Jaime (2013a). Marco Conceptual de la Auditoría Integral (primera edición). Editorial Ediloja. Ecuador.

Subía, Jaime (2013b). Auditoría de Gestión III (primera edición). Editorial Ediloja. Ecuador.

Velásquez, Marcelo (2013). Auditoría de Gestión I (primera edición). Editorial Ediloja. Ecuador.

Viloria, Norka (2004). Una aproximación a un enfoque holístico en auditoria. Revista Actualidad Contable FACES. Volumen 7, No. 9. Venezuela (Pp. 85-94). 